\title{
Effects of Infection Control Training on Dental Hygienists' Health Beliefs and Practices of Infection Control
}

\author{
Sun-Jin Moon, Kyeong-Jin Lee ${ }^{1}$, and Soo-Yeoun $\mathrm{Han}^{2 \dagger}$ \\ Dental Clinic, Kolon Couple, Jeonju 55122, \\ ${ }^{1}$ Department of Dental Hygiene, Vision College of Jeonju, Jeonju 55069, \\ ${ }^{2}$ Department of Dental Hygiene, Hyejeon College, Hongseong 32244, Korea
}

This study aims to determine the changes in health beliefs and practices of dental hygienists on infection control after having received special training on that subject. The study population consisted of dental hygienists working at the dental institutions located in Seoul and Gyeonggi areas. The intervention group and the control group each had randomly assigned 26 dental hygienists. The intervention group received training courses on infection control once a week for 3 hours, for a total of 4 training sessions. We used a survey tool to find the changes between the two groups regarding their health beliefs and practices on infection control measures. The survey was conducted prior to the training sessions, 3 months and 2 weeks after the training. The study results revealed statistically significant increases in perceived sensitivity, perceived seriousness, and perceived benefits after the training courses in the intervention group $(p<0.05)$. A statistically significant decrease in perceived barriers was also noted in the intervention group $(p<0,05)$. Additionally, there was a statistically significant increase in the practice of infection control in the intervention group as compared to the control group $(p<0.05)$. Therefore, we conclude that infection control training is crucial in bringing about positive changes to the health beliefs of dental hygienists, and a structured system is necessary for continuous infection management along with training in order to improve infection control practices.

Key Words: Dental hygienists, Education, Infection, Practice

\section{Introduction}

Consultation rooms in dental medical institutions are spaces where infection can easily arise because of environmental exposure to numerous contaminants. Contamination with patients' saliva, blood, or aerosols generated during treatments can cause cross infections among patients and dental personnel via the equipment and devices used ${ }^{1}$, and contamination by infectious disease may result due to some patients being infected with tuberculosis, herpes virus, or human immunodeficiency virus $^{2}$. In this setting, the risk of hepatitis B transmission via infected blood or saliva is reported to be 2- to 5 -fold higher than the general population ${ }^{3)}$. In order to prevent these infections, dental personnel must wash their hands thoroughly during treatment; wear personal protective equipment, such as gloves, a mask, and protective glasses; learn proper methods of disinfecting and sterilizing the devices and apparatus while properly managing supplies ${ }^{4}$; and check the overall health status and medical history of all patients visiting the clinic $^{1)}$. Dental hygienists are critical health care workers, directly providing various dental hygiene services to patients; therefore, their infection control practices have serious impact on the successor failure of dental infection control. Dental hygienists need to adopt positive attitudes and behaviors regarding infection control so that they can properly recognize the risks of infection and take appropriate 
preventive measures ${ }^{5)}$. In practice, achieving this requires changing dental hygienists' attitudes and beliefs regarding infection control ${ }^{6)}$.

Health beliefs are viewed as predictive factors for preventive and treatment behaviors against diseases, and learning from past experience and training is an important factor that can steer health beliefs and preventive behavior towards a more desirable direction ${ }^{7)}$. Therefore, in order to encourage behaviors that can effectively prevent infection, it is essential to recognize the risks, benefits, and the barriers involved in disease exposure. Since recognizing such factors promotes preventive behaviors ${ }^{8)}$, several researchers have highlighted the need for training. When were viewed the status of research on dental infection control in-country, we noted that the majority of studies focus on the current state of infection control ${ }^{9)}$, or on the awareness and implementation of certain aspects of infection prevention, or dental device disinfection and sterilization $^{9-11)}$. There have also been some studies that focused on factors relating to dental hygienists' hand-washing behavior based on the health belief model ${ }^{12)}$, factors relating to their general infection control practices, again using the health belief model ${ }^{5)}$, and the quality of infection control correlated with having received specific training ${ }^{13)}$. However, there has been lack of research on changing dental hygienists' practice after providing special training on dental infection control. Therefore, this study aimed to investigate the effects of infection control training by examining the changes in dental hygienists' health beliefs on and the practices of infection control after receiving special training.

\section{Materials and Methods}

\section{Subjects}

The subjects in this study were clinical dental hygienists working at the dental institutions in Seoul and Gyeonggi province. When Cohen's power analysis was performed with a moderate effect size of 0.80 , a significance level of 0.05 , and a power of 0.80 , the required sample size was calculated as 26 subjects per group. Based on previous studies that have effectively demonstrated real-life research situations ${ }^{14)}$, we recruited a total of 60 subjects, with the idea of assigning 30 subjects each to both the intervention and control groups, to offset the dropout rate during the training. After recruiting 60 subjects who had understood the aims of the study and voluntarily consented to participate, 30 subjects were randomly assigned to each group. After excluding 8 subjects who either did not participate in all of the sessions or did not properly complete all of the examinations, there were a total of 52 subjects remaining with 26 in the intervention group and 26 in the control group.

This study was conducted with the approval of the Wonkwang University Institution Review Board (WKIRB no. 201510-SB-040).

\section{Research instruments and procedure}

\section{1) Health beliefs}

In order to examine health beliefs on infection control, we adapted a questionnaire so that it could be administered to dental hygienists ${ }^{5)}$. After excluding preventive behavior intention from the original instrument, our questionnaire consisted of a total of 22 questions with 6 questions on perceive sensitivity, 5 questions on perceived severity, 6 questions on perceived benefit, and 5 questions on perceived barriers. Since preventive behavior intention is part of the infection control practice, preventive behavior intention was included in practice to account for its correlation with infection control practice. Each question was scored in a 5-point Likert scale where 5 points meant "strongly agree," 4 points meant "agree," 3 points meant "not sure," 2 points meant "disagree," and 1 point meant “strongly disagree." Cronbach's $\alpha$ was 0.754 for perceived sensitivity, 0.648 for perceived severity, 0.736 for perceived benefit, and 0.606 for perceived barriers.

\section{2) Infection control practice}

To measure infection control practice, we adapted and supplemented a previously studied questionnaire that measured conversion from proper awareness of infection prevention to actual implementation in the treatment room $^{15-18)}$. The questionnaire consisted of 8 questions on hand-washing, 20 questions on personal protection, 6 questions on disinfection and sterilization, and 4 questions 
on waste material management. Each question was scored on a 5-point Likert scale, where 5 points meant 'always,' 4 points meant 'often,' 3 points meant 'sometimes,' 2 points meant' rarely,' and 1 point meant 'never.' Cronbach's $\alpha$ for the questionnaire was 0.680 .

\section{3) Infection control training}

We included in the training materials the recommended content for infection control standards from the U.S. Centers for Disease Control and Prevention (CDC) Guidelines ${ }^{19)}$, and used the teaching plans developed for the dental infection controller certification courses operated by the Korean Society of Dental Equipment Administrators. We sought counsel from 2 professors of dental hygiene to confirm that the educational content was appropriate for our purposes. Apart from the sections on devices and equipment, we devised a training plan using the sections that related to the treatment room.

The intervention group participated in infection control training a total of 4 times once a week in 24th October 14th November 2015. Each session was 3 hours. The length of this training was determined to be 4 sessions in total based on the outcome of the training on infection control for nursing students ${ }^{14)}$, and the sessions were conducted in groups of $5 \sim 6$ persons. During that time, the control group did not receive any training. The training was conducted in collaboration with the Korean Society of Dental Equipment Administrators; a dental infection control expert, currently conducting dental infection control lectures and practical classes and an assistant researcher also participated. To increase understanding and interest, the training was conducted at a dental institution in Sinjeong-dong, Yangcheon-gu, Seoul, which was commonly used for theoretical and practical trainings.

In order to compare changes in health beliefs and infection control practice in the intervention group who received specialized training and the control group who did not receive this training, identical questionnaires were distributed pre-education, 2 weeks and 3 months post-education, and the mean results were compared.

\section{Data analysis \\ PASW Statistics ver. 18.0 (IBM Co., Armonk, NY, USA)}

was used to analyze the data in accordance with the research objectives. Questions on subjects' general characteristics and infection-related characteristics were examined by frequency analysis. A repeated measure analysis of variance (RM-ANOVA) was performed to identify changes in health beliefs and infection control practice after the training. Cross-tabulation analysis was performed to verify homogeneity between the intervention group and the control group. A significance level of $\mathrm{p}<0.05$ was used for all statistical tests.

\section{Results}

\section{Subjects' general characteristics and homogeneity}

The subjects' general characteristics are displayed in Table 1. The predominant age group in both the intervention and the control group was $20 \sim 29$ years of age (17 individuals). Sixteen subjects in the intervention group and 17 subjects in the control group had 6 years or less work experience. The type of workplace was 'dental clinic' for 21 individuals in the intervention group and 22 individuals in the control group, meaning that the majority of individuals were working at the primary hospitals.

Table 1. General Characteristics of Subjects $(n=26)$

\begin{tabular}{lcccc}
\hline Characteristics & $\begin{array}{c}\text { Study } \\
\text { group }\end{array}$ & $\begin{array}{c}\text { Control } \\
\text { group }\end{array}$ & $\chi^{2}$ & p-value \\
\hline Age $(\mathrm{y})$ & & & 1.059 & 1.000 \\
$20 \sim 29$ & $17(65.4)$ & $17(65.4)$ & & \\
$30 \sim 39$ & $8(30.8)$ & $9(34.6)$ & & \\
$40 \sim 49$ & $1(3.8)$ & 0 & & \\
Final education & & & 0.758 & 0.697 \\
College & $15(57.7)$ & $18(69.2)$ & & \\
University & $8(30.8)$ & $6(23.1)$ & & \\
Graduate school & $3(11.5)$ & $2(7.7)$ & & \\
Work experience $(y)$ & & & 0.820 & 0.891 \\
$1 \sim 3$ & $9(34.6)$ & $8(30.8)$ & & \\
$4 \sim 6$ & $7(26.9)$ & $9(34.6)$ & & \\
$7 \sim 9$ & $6(23.1)$ & $4(15.4)$ & & \\
$\geq 10$ & $4(15.4)$ & $5(19.2)$ & & \\
Work organization & & & 0.223 & 1.000 \\
Dental clinic & $21(80.8)$ & $22(84.6)$ & & \\
Dental hospital & $3(11.5)$ & $2(7.7)$ & & \\
Etc. & $2(7.7)$ & $2(7.7)$ & & \\
\hline
\end{tabular}

Values are presented as $\mathrm{n}(\%)$.

The data were analysed chi-square test, Fisher exact test. 
Homogeneity testing revealed no significant differences in general characteristics between the two groups.

\section{Changes in health beliefs following infection control training}

The RM-ANOVA showed statistically significant effects of group and time $(\mathrm{p}<0.05)$, as well as the interaction between group and time $(\mathrm{p}<0.05)$ for perceived sensitivity. No statistically significant effect of group $(p>0.05)$ was noted for perceived severity, but there was a statistically significant effect of time $(p<0.05)$, and of the interaction between group and time $(\mathrm{p}<0.05)$.

Regarding perceived benefit, there was no statistically significant effect between groups ( $p>0.05)$, but there was a statistically significant effect of time $(p<0.05)$, and of the interaction between groups and time $(p<0.05)$. There were statistically significant effects of group and time $(p<$
0.05) for perceived barriers as well as the interaction between groups and time $(\mathrm{p}<0.05)$ (Table 2$)$.

\section{Changes in infection control practice following the infection control training}

Table 3 shows the results of the RM-ANOVA for dental hygienists' infection control practice. There were statistically significant effects of group and time $(p<0.05)$ as well as the interaction between groups and time $(\mathrm{p}<0.05)$.

\section{Discussion}

Infection control training can be provided through regular or supplementary courses at educational and public institutions, or through training courses at the designated institutions. We believe that knowledge should form the basis for the actual practice. Therefore, in our

Table 2. Change of Health Belief according to Infection Control Education

\begin{tabular}{|c|c|c|c|c|c|}
\hline Category & Study group & Control group & Source & $\mathrm{F}$ & $\mathrm{p}$ \\
\hline \multicolumn{6}{|c|}{ Perceived sensitivity } \\
\hline Baseline & $3.46 \pm 0.36$ & $3.44 \pm 0.34$ & Group & 12.685 & 0.001 \\
\hline $2 \mathrm{wk}$ & $3.90 \pm 0.21$ & $3.40 \pm 0.43$ & Time & 17.842 & $<0.001$ \\
\hline $3 \mathrm{mo}$ & $3.79 \pm 0.20$ & $3.46 \pm 0.36$ & Group $\times$ time & 20.39 & $<0.001$ \\
\hline \multicolumn{6}{|c|}{ Perceived severity } \\
\hline Baseline & $2.62 \pm 0.28$ & $2.82 \pm 0.43$ & Group & 0.296 & 0.589 \\
\hline $2 \mathrm{wk}$ & $2.95 \pm 0.30$ & $2.68 \pm 0.37$ & Time & 7.317 & 0.001 \\
\hline $3 \mathrm{mo}$ & $2.94 \pm 0.34$ & $2.87 \pm 0.38$ & Group $\times$ time & 11.059 & $<0.001$ \\
\hline \multicolumn{6}{|c|}{ Perceived benefit } \\
\hline Baseline & $3.43 \pm 0.29$ & $3.56 \pm 0.33$ & Group & 0.911 & 0.344 \\
\hline $2 \mathrm{wk}$ & $3.89 \pm 0.15$ & $3.76 \pm 0.19$ & Time & 39.999 & $<0.001$ \\
\hline $3 \mathrm{mo}$ & $3.86 \pm 0.15$ & $3.74 \pm 0.16$ & Group $\times$ time & 6.723 & 0.002 \\
\hline \multicolumn{6}{|c|}{ Perceived disability } \\
\hline Baseline & $3.52 \pm 0.36$ & $3.55 \pm 0.38$ & Group & 7.797 & 0.007 \\
\hline $2 \mathrm{wk}$ & $3.32 \pm 0.42$ & $3.64 \pm 0.52$ & Time & 9.799 & $<0.001$ \\
\hline $3 \mathrm{mo}$ & $3.10 \pm 0.35$ & $3.54 \pm 0.34$ & Group $\times$ time & 8.295 & 0.002 \\
\hline
\end{tabular}

Values are presented as mean \pm standard deviation.

The data were analyzed repeated measure ANOVA.

Table 3. Change of Infection Control Performance according to Infection Control Education

\begin{tabular}{lcclrc}
\hline \multicolumn{1}{c}{ Category } & Study group & Control group & Source & F & p \\
\hline Baseline & $2.58 \pm 0.17$ & $2.49 \pm 0.16$ & Group & 163.846 & $<0.001$ \\
$2 \mathrm{wk}$ & $3.31 \pm 0.11$ & $2.53 \pm 0.18$ & Time & 91.568 & $<0.001$ \\
$3 \mathrm{mo}$ & $2.93 \pm 0.21$ & $2.50 \pm 0.17$ & Group $\times$ time & 73.985 & $<0.001$ \\
\hline
\end{tabular}

Values are presented as mean \pm standard deviation.

The data were analyzed repeated measure ANOVA. 
study, we aimed to ascertain dental hygienists' characteristics relating to infection within the treatment room, and to examine changes in their health beliefs about infection and infection control practice after having received the infection control training. We found that perceived sensitivity increased significantly after the infection control training, and this finding corroborates with the observation of increased perceived sensitivity among nurses who received training on blood-mediated infection control in a previous study ${ }^{6}$. The results are also consistent with another study that reported increased perceived risk in nursing students after receiving blood-mediated infection prevention education ${ }^{20)}$, and a study reporting an increase in perceived risk of AIDS in medical personnel after describing the risks of blood-related infectious disease and the extent of disease prevention $^{21)}$. This emphasizes the effect of implementing practical training alongside theoretical instruction.

Perceived severity increased significantly after the infection control training, and this is consistent with a study reporting a significant increase in perceived severity among nurses who underwent blood-mediated infection control education"), and a study showing a significant difference in perceived severity, among a group of female students who had training on inoculation against human papilloma virus as compared with the control group ${ }^{22)}$. This significant increase in perceived sensitivity and perceived severity following the training on dental infection control is believed to be very important in terms of effective motivation for the actual practice of infection control.

Perceived benefit increased significantly after the training in the intervention group as compared to the control group. This is consistent with the previous studies that have shown perceived benefit to be an important factor that can promote actual implementation easier than the other health belief model components ${ }^{23)}$, and that perceived benefit is effective when used in the development of educational programs ${ }^{24)}$, and that infection control practice increases when perceived benefit increases $^{12)}$. Thus, it is believed that the increase in perceived benefit with training was an important factor directly affecting implementation, and that it was the most significant factor in increasing better infection control practice.

In terms of perceived barriers, previous studies have reported that it is difficult to reduce perceived barriers with short-term training, and a systematic training program should be developed ${ }^{25}$. However, the 4-week-long training provided in our study produced a significant decrease in perceived barriers. This supports a previous study that reported the need to give sufficient time for an educational intervention to generate positive changes ${ }^{24)}$. Moreover, studies with nurses and nursing students were able to produce significant changes in perceived barriers following a blood-mediated infection control program ${ }^{5,22)}$, and a study implementing health education for the rheumatoid arthritis patients ${ }^{26}$ also reported a decrease in perceived barriers. Therefore, the significant decrease in perceived barriers in the present study is believed to be the result of implementing a program based on perceived sensitivity, using theory and practice.

Infection control practice increased significantly in the intervention group as compared to the control group in our study. The largest increase was from pre-education to 2 weeks post-education; and although there was a slight decrease after 3 months post-education, the level of practice was still higher than that of pre-education. Thus, we confirmed that the training on dental infection control had an effect that persisted at least 3 months. A previous study on infection control practice among nurses reported a continuing increase at 2 weeks and 3 months post-training when compared with pre-training ${ }^{27)}$. This is similar to our finding that the effect at 3 months post-training remained higher than the pre-training levels. To improve infection control practice, continued feedback from infection monitoring data is essential in addition to training ${ }^{5)}$. The fact that we achieved a significant increase in infection control practice is believed to be due to individuals in the intervention group received active feedback from their instructors during the training period, as well as the effects of the teaching plan that focused on content that could actually be applied to clinical practice. Developing and implementing simulation-based infection control training are effective in improving the actual infection control practice $^{8)}$, and a study implementing simulation-based 
education for nursing students found that, compared to a group that received only theoretical training, the group that had training in an environment imitating real-life situation showed a greater improvement in infection control practice and clinical work performance. Simulation-based training is an effective method for improving practice, and we expect this to also be true for training the dental hygienists on infection control.

This study examined the changes in dental hygienists' health beliefs and infection control practice after receiving a special training on dental infection control, and demonstrated the effects of such training. Because a similar study with dental hygienists has not been attempted so far in Korea, it is not possible for us to compare our results with any previous research. This study did have some limitations. Dental hygienists' infection control practice was measured by a questionnaire rather than measuring directly on-site, and we were not able to examine changes in the effects of health beliefs on infection control practice after the training. Nevertheless, based on the results of the previous studies involving other health care professionals, the training on dental infection control in our study was conducted on-site at a dental institution, and consisted of both theoretical and practical training under conditions similar to a real world clinical, working environment. Because our education plan was based on content that was actually used in clinical situations, and it was already in the curriculum for dental infection controller certification, it provided accurate methods of infection control that could be used directly to train dental hygienists. We anticipate that the training on dental infection control described in this study will improve infection control practice and contribute to the development of future training programs. However, preventing infections in dental institutions requires continued assessment of the outcome of the training, development of standardized training resources systematic programs that involve continuous education with diverse training methods suited to the level of dental institution, and lastly, institutional support for these programs. Future research will be required to investigate how changes in health beliefs affect changes in the infection control practice.

\section{References}

1. Kim HR, Park MR, Sung HJ, Cho CY, Roo HK: Perception and implementation for dental infection control in Busan, Kyungnam areas. J Korean Acad Dent Hyg 14: 11-21, 2012.

2. Kim KS, Kim KM, Kwon HM, et al.: Safety standards and infection control for dental hygienists. 2nd ed. KMS, Seoul, pp.14-19, 2010.

3. Oh SK: A practice of infection control in dental office. J Korean Dent Assoc 33: 184-187, 1995.

4. Kim JH, Lee KY: A study on the infection control attitude of dental hygienists. J Dent Hyg Sci 9: 129-136, 2009.

5. Hong SH: The associated factors with the performance of infection control based on the health belief model among the dental hygienists in dental clinics. Unpublished doctoral dissertation, Chosun University, Gwangju, 2014.

6. Choi JS, Kim KS: Application and evaluation of a web-based education program on blood-borne infection control for nurses. J Korean Acad Nurs 39: 298-309, 2009.

7. Shin SJ, Shin KR, Yi HR, Ju SK: Knowledge, health belief, and self-efficacy related to osteoporosis. J Korean Acad Nurs 35: 850-857, 2005.

8. Ramsey PW, Glenn LL: Nurses' body fluid exposure reporting, HIV testing, and hepatitis B vaccination rates: before and after implementing universal precautions regulations. AAOHN J 44: 129-137, 1996.

9. Cho YJ, Yoon SJ, Ahn HS, Kim SD, Park HK: A study on infection control practices among dental hygienists. J Korean Soc Qual Assur Health Care 10: 190-203, 2003.

10. Yoon MS, Hong YP, Jung YK: The investigation on infection control in dental clinics. Nurs Sci Res Inst 1: 95-141, 1997.

11. Nam YS, Park MS: A study on practice level of infection prevention of dental hygienists in dental clinics. J Korean Acad Oral Health 34: 187-197, 2010.

12. Lim MH: Influential factors related to hand washing practice of dental hygienists by health belief mode. J Korean Soc Dent Hyg 13: 193-200, 2013.

13. Kim JH, Kim JK: Infection control among dental hygienists according to infection control education experiences. J Korean Soc Dent Hyg 11: 547-556, 2011.

14. Kim SH: Development and effects of a simulation-based education program for healthcare-associated infection control for nursing students. Unpublished doctoral dissertation, 
Kyungpook National University, Daegu, 2016.

15. Yoon KO: Actual status of infection control by the dental hygienist in Korea. J Korean Soc Dent Hyg 13: 369-376, 2013.

16. Park HS, Bae JY, Lee YA, Jo MJ: A study on recognition of infection control among dental staff. J Dent Hyg Sci 7: 257-262, 2007.

17. Kohn WG, Collins AS, Cleveland JL, et al.: Guidelines for infection control in dental health-care settings--2003. MMWR Recomm Rep 52: 1-61, 2003.

18. Wang H, Fennie K, He G, Burgess J, William AB: A training programme for prevention of occupational exposure to bloodborne pathogens: impact on knowledge, behaviour and incidence of needle stick injuries among student nurses in Changsha, people's republic of China. J Adv Nurs 41: 187-194, 2003.

19. Hentgen V, Jaureguiberry S, Ramiliarisoa A, Andrianantoandro V, Belec M: [Knowledge, attitude and practices of health personnel with regard to HIV/AIDS in Tamatave (Madagascar)]. Bull Soc Pathol Exot 95: 103-108, 2002. French.

20. Lee EJ, Kim HO: Effects of human papillomavirus vaccination education on college women's knowledge, health belief, and preventive behavior intention. J Korean Acad Nurs 42: 715-723, 2011.
21. Jang JY: The correlation study on oral health behavior toward oral health belief. J Korean Acad Dent Hyg 9: 133-148, 2007.

22. Kim HJ, Yang SJ: The effects of education on the prevention of noise-induced hearing loss in adolescence. J Korean Public Health Nurs 27: 357-371, 2013.

23. Yoon IK, Jang MH, Kim GT, et al.: Development and application of health belief model based milk education program for elementary, middle, and high school students. J Korean Home Econ Educ Assoc 19: 17-36, 2007.

24. Lee EN: The effect of the health belief and efficacy expectation promoting program on osteoporosis preventive health behavior in women with rheumatoid arthritis. J Muscle Jt Health 5: 174-190, 1998.

25. Kim KS, Lee SM: Effects of ventilator-associated pneumonia (VAP) control education on ICU nurses' infection control performance and incidence of pneumonia. J Korean Crit Care Nurs 3: 13-22, 2010.

26. Cho SS, Kim KM, Lee BY, Park SA: The effects of simulation-based infection control training on the intensive care unit nurses' perception, clinical performance, and self-efficacy of infection control. J Korean Clin Nurs Res 18: 381-390, 2012.

27. Cant RP, Cooper SJ: Simulation-based learning in nurse education: systematic review. J Adv Nurs 66: 3-15, 2010. 